\title{
A PROV Encoding for Provenance Analysis Using Deductive Rules
}

\author{
Paolo Missier ${ }^{1}$ and Khalid Belhajjame ${ }^{2}$ \\ 1 Newcastle University, \\ Newcastle upon Tyne, UK \\ Paolo.Missier@ncl.ac.uk \\ 2 University of Manchester \\ Oxford Road, Manchester, UK \\ Khalid.Belhajjame@cs.man.ac.uk
}

\begin{abstract}
PROV is a specification, promoted by the World Wide Web consortium, for recording the provenance of web resources. It includes a schema, consistency constraints and inference rules on the schema, and a language for recording provenance facts. In this paper we describe a implementation of PROV that is based on the DLV Datalog engine. We argue that the deductive databases paradigm, which underpins the Datalog model, is a natural choice for expressing at the same time (i) the intensional features of the provenance model, namely its consistency constraints and inference rules, (ii) its extensional features, i.e., sets of provenance facts (called a provenance graph), and (iii) declarative recursive queries on the graph. The deductive and constraint solving capability of DLV can be used to validate a graph against the constraints, and to derive new provenance facts. We provide an encoding of the PROV rules as Datalog rules and constraints, and illustrate the use of deductive capabilities both for queries and for constraint validation, namely to detect inconsistencies in the graphs. The DLV code along with a parser to map the PROV assertion language to Datalog syntax, are publicly available.
\end{abstract}

\section{Introduction}

Work towards standardization of a model for expressing the provenance of Web resources has been in progress at the W3C since 2011. The outcome of this community effort comprises (i) a conceptual data model (PROV-DM) [Mor12a], (ii) a set of consistency constraints on the model (PROV-C) Mor12b], (iii) a formal notation (PROV-N) Mor12c], and, in the near future, a set-theoretical semantic:1. Consequently, implementations of the PROV specification should include a parser for the language, a constraint analyzer, and a query model (language and processor) to match the data model. In this paper we propose Datalog as a natural choice of programming model for a PROV implementation that fits these requirements. Rooted in first-order logic, Datalog has been popular amongst the

\footnotetext{
${ }^{1}$ All of these components of the specification are still in progress at the time of writing.

P. Groth and J. Frew (Eds.): IPAW 2012, LNCS 7525, pp. 67-81, 2012.

(C) Springer-Verlag Berlin Heidelberg 2012
} 
data management community for a very long time, well past its heyday as a foundation of deductive database theory CGT90, mostly due to its expressiveness in capturing formal properties of queries and query rewriting methods [Hal01]. Datalog implementations are becoming popular again, thanks in part to the current momentum around Answer Set Programming [BET11, a model for declarative problem solving that is closely related to the Datalog model. Indeed, our prototype implementation is based on DLV2, a deductive database system based on disjunctive Datalog [EGM97] with additional constraint-solving capabilities.

\subsection{Contributions and Approach}

Our contribution is threefold. Firstly, we provide a (nearly) complete mapping of PROV constraints to Datalog rules 3 , and elaborate on the cases where such mapping is not appropriate. Secondly, we illustrate the expressive power of declarative rules in expressing significant examples of provenance graph queries, highlighting the natural fit of recursive rules to graph traversal. Finally, we apply DLV constraint checking to the problem of validating provenance graphs, for instance to determine temporal consistency.

Our approach involves (i) translating provenance expressed in the native PROV notation into a Datalog database, (ii) encoding PROV constraints as Datalog rules and constraints, and (iii) extending the core set of rules to express specific query patterns on provenance graphs. The resulting prototype implementation is available online 4 .

\section{$1.2 \quad$ Related Work}

Datalog has a long history as a theoretical tool in data management. Specifically, in data integration, Datalog has been commonly used as a notation to analyze and compare existing query rewriting algorithms Hal01, where recursive Datalog rewritings are essential. Shen et al. [SDNR07] used Datalog rules as a means of developing information extraction programs. Compared with similar techniques that use low-level programming languages such as Perl, $\mathrm{C}++$ or Java, Datalog offers a declarative style along with a powerful mechanism for composing modules, which can be written by multiple users, into larger programs. Data$\log$ has also been used in data exchange to specify schema mappings between heterogeneous schemas ABR10, and in model translation to transform schemas from one model to another, for example from Relational to XML [ACG07.

Datalog has also been used in workflow provenance literature by a handful of researchers, typically as an illustration of queries at a conceptual level $\left[\mathrm{ABC}^{+} 10\right.$. For example, Cohen et al. CBD06] chose Datalog as a notation for formally

\footnotetext{
2 http://www.dlvsystem.com/

3 This mapping reflects the state of the PROV constraints as of June, 2012. Up-to-date versions of the implementation are maintained online, as indicated here below.

4 at gitHub: http://bit.ly/HOY15T (code and examples), http://bit.ly/HOYJA8
} (PROV-N to Datalog parser), see credits at the end of the paper. 
defining provenance views that take into account the chained and nested structure of scientific workflows. We note, however, that the role of Datalog in such proposal is confined to a notation to illustrate provenance queries, as opposed to an actual query language. In this respect, the proposal by Dey et al. [DZL11] is closer to our work, in the sense that they use Datalog rules at the core of their ProPub system for policy-driven selection of public provenance fragments. This is similar in spirit to our approach, which however is focused on the comprehensive encoding of a set of rules and constraints that are prescribed by a standard specification. Additionally, we highlight the potential of the constraint-solving capabilities of the DLV implementation of disjunctive Datalog, which we argue, will play an important role in provenance analysis and validation.

\section{PROV Provenance Graphs as Deductive Databases}

We begin by providing an overview of the PROV provenance model by means of an example, and show how it can be encoded as extensional Datalog programs in a natural way.

\subsection{Example: Collaborative Document Editing}

The example presents an account of how a document was collaboratively edited and published by a group of co-authors, led by Alice and including Bob and Charlif 5 . Bob has produced the initial draft-v1, which includes references to two papers, paper1 and paper2. Alice then left some comments in document draft-comments, including the recommendation to also consider paper 3 in the next revision. Bob then used the comments to produce draft-v2. At this point Charlie, who like Bob works for Alice, published the document as Working Draft WD1, using the publication guidelines pub-guide-v1 issued by the W3C. He, however, ignored version pub-guide-v2 of those guidelines, which the W3C had issued as update before the publication process was completed.

A graph depiction of this account of events is shown in Fig. 1 using a non-prescriptive graphical notation. Three types of nodes appear in the graph, namely entities, activities, and agents, and arcs represent directed associations amongst these elements. Node types and their associations are all part of the PROV specification. Notable relations used in the example include the usage (used) of an entity by an activity, the generation (wasGeneratedBy) of a new entity by an activity, the derivation of an entity from another (wasDerivedFrom), the responsibility of an agent for an activity (wasAssociatedWith), and a "chain of responsibility" relation, actedOnBehalfOf. Note also that the publication activity pub involves the additional plan pub-guide-v1.

The graph also illustrates a more subtle point, namely that Bob was aware of paper3, although the paper itself was not "consumed" as part of the editing activity. This is achieved by introducing two entities, Bob-1 and Bob-2, both

${ }^{5}$ This example is modified version of one that appeared in early versions of the PROV specification draft, and is used with permission from the editors. 


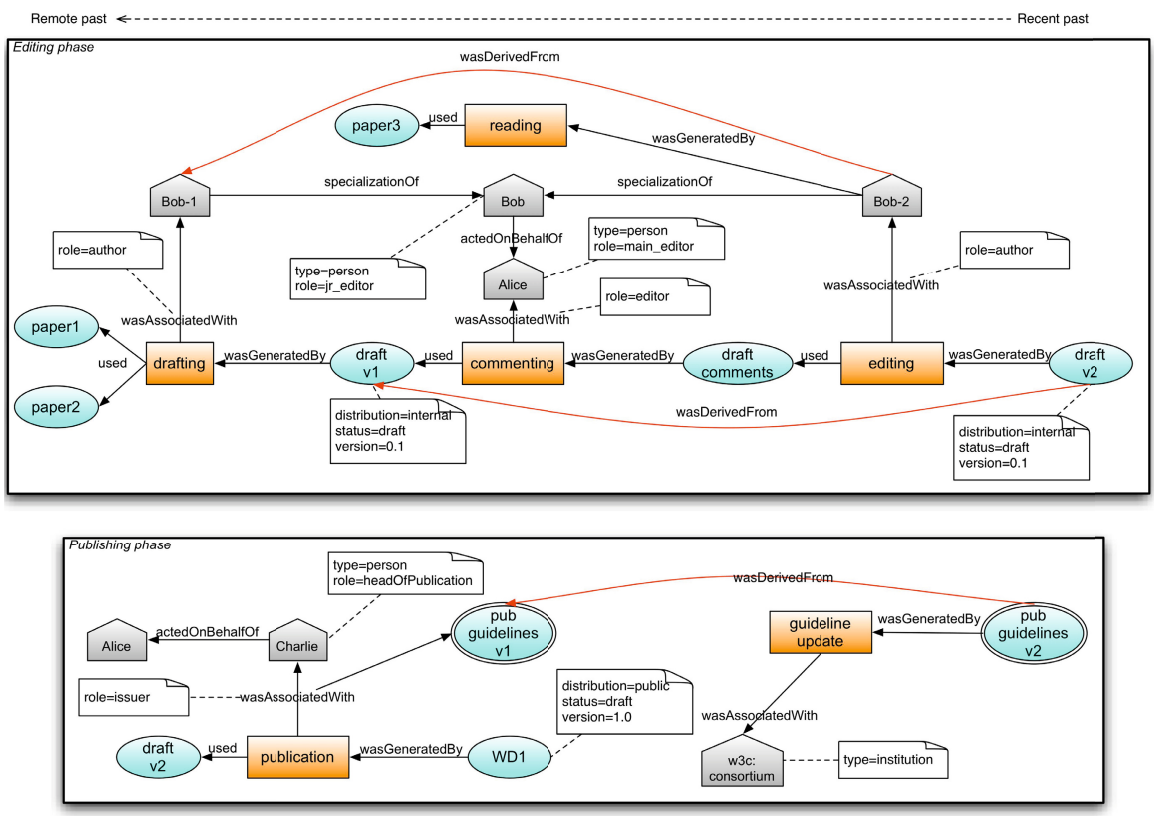

Fig. 1. PROV provenance graph for collaborative document editing

of which specialize the more generic Bob. The reading activity used paper3 and thus accounts for Bob-2 being "derived from" Bob-1. Thus, Bob-1 and Bob-2 describe two states of the same person, Bob.

The general idea behind PROV is that the graph of relations embodies the provenance of its entities, for example the genesis of WD1 is obtained by traversing the graph in the direction of the associations (from the "recent past" back to the "remote past"), or by querying the graph, for example "who was responsible for the comments"? Examples of traversal and queries on provenance graphs are presented throughout the rest of the paper.

\subsection{Background: Datalog Basics}

In order to make the paper as self-contained as possible, we now briefly recall the basics of the Datalog model. A complete account of Datalog can be found in the classic paper, CGT89. A Datalog program consists of a set of rules, which are expressions of the form

$$
L_{0}:-L_{1}, \ldots, L_{n}
$$

where the $L_{i}$ are literals, i.e., either positive or negated atoms, of the form $p\left(t_{1}, \ldots, t_{k}\right)$ where $p$ is a predicate symbol and the terms $t_{i}$ are either constants or variables. $L_{0}$ is the head of the rule, the remaining $L_{i}$ form the body of the rule. A ground literal is one that contains only constants. Rules with a ground 
literal in the head and empty body are ground facts. The set of ground facts is referred to as the Extensional Database (EDB), in contrast to the Intensional Database (IDB) consisting of rules with non-empty body.

The purpose of rules is to derive new facts from existing ones. For example, the rule:

entity $(X, \operatorname{Attrs}):-\operatorname{agent}(X, \operatorname{Attrs})$.

(by convention, terms in upper case denote variables) allows for a new fact entity ( $\mathrm{x}$, attrs) to be derived, where $\mathrm{x}$ and attrs are constants, provided there is a substitution of variables $\Theta=\{X / \mathrm{x}$, Attrs/attrs $\}$ such that $\Theta(\operatorname{agent}(X, A t t r s))$ is a ground fact (either in the EDB or itself derived). In our example, the result includes all the agents, i.e. \{ entity(alice,alice_attrs), entity (bob, bob_attrs),...\}. In a sense, this rule simply defines "specialization" and has the effect of adding all agents to the EDB as new entities.

In general, a substitution $\Theta$ satisfies the body of a rule (10) if the database contains all of the ground literals $\Theta\left(L_{i}\right)$. If $\Theta$ satisfies the body, the new ground literal $\Theta\left(L_{0}\right)$ is added to the database. Note that $\Theta$ must substitute for all variables in the head. This is guaranteed by requiring that the rule be safe, i.e., that all variables that appear in the head also appear in the body of the rule.

\subsection{Datalog Encoding of PROV Graphs}

The PROV specification includes a formal notation, called PROV-N, for expressing PROV graphs. Its syntax is so close to that of Datalog ground facts, that we can safely present fragments of the example above using the latter, with nearly no loss of information, with a few exceptions as noted below. For example, the following PROV-N fragment states the existence of entity draftV1, activity drafting with a start and end time, and the generation of draftV1 by the drafting activity.

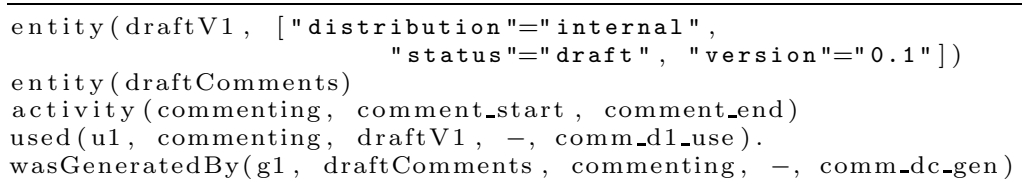

The first entity is qualified with an optional set of attributes. Start and end events can optionally be specified, e.g. for activity, usage (comm_d1_use denotes the time draftV1 begins to be used), and generation (i.e., comm_dc_gen the time at which draftComments is complete). In PROV-N, the '-' symbol indicates a null, or unavailable value. $\mathrm{u} 1$ and $\mathrm{g} 1$ are identifiers for the use and generation relations, respectively, and can be referenced from other relations.

\footnotetext{
${ }^{6}$ The safety property includes the additional condition that each variable in the body appears in at least one positive literal.
} 
Below is the set of Datalog ground facts for the same fragment:

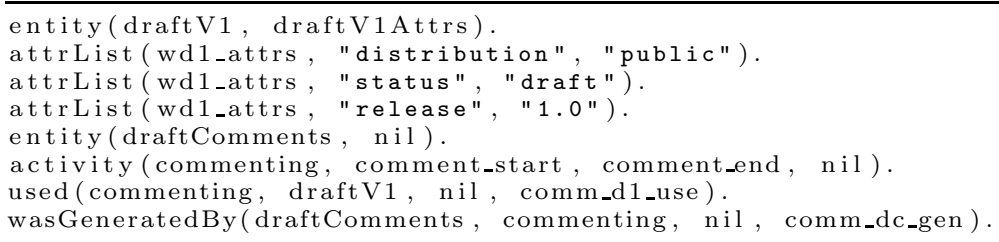

In the mapping from PROV to Datalog, the relation names become predicate names and the relation arguments simply become terms. However, there are a few differences. Firstly, lists of attributes are mapped to a separate predicate symbol attrList and linked to their parent element by means of a new identifier, i.e., draftV1Attrs. This makes it easy to write queries that involve attributes. A Datalog query, or goal, is specified as a conjunction of literals followed by a question mark 8 , for example:

entity(draftV1s, Attrs), attrList (Attrs, Name, Value) ?

The result of the query includes all ground facts in the EDB that match all the literals in the goal, for some substitution of the variables in the goals' literals. Our example query returns:

draftV1_attrs, "distribution ", "internal"

draftV1_attrs, "status", "draft"

The second difference is that, while in PROV events can be expressed using timestamps, these cannot be used for temporal reasoning, because there is no assumption that they will have been generated by the same clock. Rather, what matters for provenance consistency is only the partial order amongst events. This justifies choosing purely symbolic terms in these examples.

Finally, in this "baseline" mapping we choose not to create new identifiers (which could be done by means of Skolem functions). As a consequence, each new relation created by means of a rule cannot be given an identifier, for example, the following rule generatea a new used relation from the presence of others in the EDB:

\begin{tabular}{rrr}
\hline used(A,E1, nil , Attrs $)$ & $:-$ wasDerivedFrom (E2, E1, - , Attrs ), \\
& wasGeneratedBy( E2, A, Attrs, - ).
\end{tabular}

Since relation identifiers are optional in PROV, in our implementation we choose not to use them.

For reference, additional excerpts of the Datalog EDB for our running example can be found in Appendix $\mathrm{A}$

\section{PROV Constraints as Datalog Rules}

In this section we present a selected set of rules that encode PROV constraints, and show them at work on example queries that are relevant for provenance graph analysis.

\footnotetext{
7 The null symbol "-" is not legal in Datalog, and is replaced by "nil".

8 The question mark at the end is DLV-specific syntax.
} 


\subsection{Mapping PROV Rules to Datalog Rules and Queries}

For the most part, PROV rules are of the form if $r_{1}, \ldots, r_{n}$ then $r$, where the antecedents $r_{i}$ are relations in the provenance graph, and the consequent $r$ is a new relation. For example: "If wasDerivedFrom(e2,e1,a,g2,u1) holds, for some a, g2, u1, then tracedTo $(\mathrm{e} 2, \mathrm{e} 1)$ also holds." 9 (in some cases, the rule specifies both sufficient and necessary conditions for $r$ ). In general, there is a natural mapping of these rules to Datalog, which involves creating a deductive rule with head $r$ and body $r_{1} \ldots r_{n}$ with suitable variables. For example, the entire set of traceability constraints is encoded in Datalog as follows 10 :

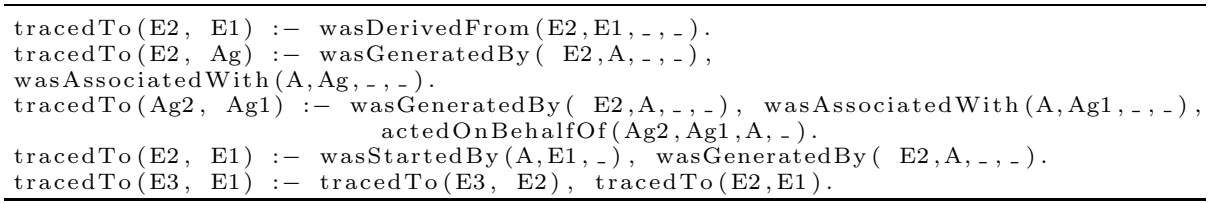

The first rule states that if entity E2 was derived from E1, then it is also true that E2 can be traced to E1. Rule (2) states that entity E2 can be traced to agent $\mathrm{Ag}$ if $\mathrm{E} 2$ was generated by an activity $\mathrm{A}$, and $\mathrm{Ag}$ was associated with (i.e., was responsible for) A. The last rule states transitivity.

In relational database terms, rules define views over the EDB, which can be used to derive a new set of database facts, in this case of the form tracedTo ( 1 , e2). Given the rules, the simple query computes the "traceability" grap induced by the EDB given the rules:

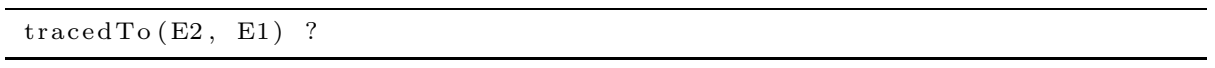

The set of substitutions for E2, E1 returned by DLV in response to the query are shown in Table 1, along with an explanation for their derivation (note that one can ask more specific queries where some of the terms are ground, for example tracedTo (draftV2,E)? for "what is draftV2 traced to?").

\subsection{Limitations of Mapping and Rules as DLV Constraints}

We have seen that rules are used to deduce new ground literals from existing ones. These literals, however, can only contain terms that appear in the body of the rule. We do not map rules that require the introduction of new constants that represent new nodes in the provenance graph (this is also consistent with our earlier decision not to mint new identifiers) 11 . For example, the rule: wasRevision0f (new,old,ag) $\Rightarrow \exists e \mid$ specialization0f (new,e) is not mapped, as it entails introducing a new entity $e$ into the provenance graph.

\footnotetext{
${ }^{9}$ Quoting [Mor12a, "A traceability relation between two entities e2 and e1 is a generic dependency of e 2 on e1 that indicates either that e1 may have been necessary for e 2 to be created, or that e1 bears some responsibility for e2's existence.".

${ }^{10}$ Consistent with our earlier choice for mapping PROV relations, we ignore the relation identifiers g2 and $u 1$.

${ }^{11}$ Note however, that some of the rules introduce default values for some of the terms, typically for attribute-value pairs.
} 
Table 1. Substitutions leading to the "traceability" graph induced by the provenance graph on the running example

\begin{tabular}{|l|l|l|}
\hline substitution & rules & facts involved \\
\hline $\begin{array}{l}\text { E2/draftV1, } \\
\text { E1/bob_1 }\end{array}$ & $(2)$ & $\begin{array}{l}\text { wasGeneratedBy(draftV1, drafting, nil, nil), } \\
\text { wasAssociatedWith(drafting, bob_1, nil, waw1_attrs). }\end{array}$ \\
\hline $\begin{array}{l}\text { E2/draftComments, } \\
\text { E1/alice }\end{array}$ & $(2)$ & $\begin{array}{l}\text { wasGeneratedBy(draftComments, commenting, nil, comm_dc_gen). } \\
\text { wasAssociatedWith(commenting, alice, nil, waw2_attrs). }\end{array}$ \\
\hline $\begin{array}{l}\text { E2/draftV2, } \\
\text { E1/draftV1 }\end{array}$ & $(1)$ & wasDerivedFrom(draftV2, draftV1, nil, nil) \\
\hline $\begin{array}{l}\text { E2/draftV2, } \\
\text { E1/bob_2 }\end{array}$ & $(2)$ & $\begin{array}{l}\text { wasGeneratedBy(draftV2, editing, nil, edit_d2_gen). } \\
\text { wasAssociatedWith(editing, bob_2, nil, waw3_attrs). }\end{array}$ \\
\hline $\begin{array}{l}\text { E2/draftV2, } \\
\text { E1/bob_1 }\end{array}$ & $\begin{array}{l}(1), \quad(2), \\
\text { wasGeneratedBy(draftV2, editing, nil, edit_d2_gen). } \\
\text { wasAssociatedWith(editing, bob_2, nil, waw3_attrs). } \\
\text { wasDerivedFrom( bob_2, bob_1, nil, nil). }\end{array}$ \\
\hline $\begin{array}{l}\text { E2/pubGuidelinesV2, } \\
\text { E1/pubGuidelinesV1 }\end{array}$ & $(1)$ & wasDerivedFrom(pubGuidelinesV2, pubGuidelinesV1, nil, nil). \\
\hline E2/wd1, E1/charlie & $(2)$ & $\begin{array}{l}\text { wasGeneratedBy(wd1, publication, nil, pub_wd1_gen). } \\
\text { wasAssociatedWith(publication, charlie, pubGuidelinesV1, nil). }\end{array}$ \\
\hline E2/bob_2, E1/bob_1 & $(1)$ & wasDerivedFrom(bob_2, bob_1, nil, nil). \\
\hline
\end{tabular}

Also, a few PROV rules either cannot be captured as Datalog rules, or otherwise lead to unsafety. The following PROV rule, for example, states that the alternate 0 felation is anti-symmetric, in terms of an inference that leads to a new equality amongst entities being introduced in the model:

specializationOf $(E 2, E 1) \wedge$ specializationOf $(E 1, E 2) \Rightarrow E 1==E 2$.

The new equality in the consequent is problematic, as it cannot be expressed simply by using the built-in equality predicate '=', but instead would require a new predicate, say equal/2, as in:

equal(E1,E2) :- specializationOf(E1, E2), specializationOf(E2, E1).

However, one cannot define the semantics of equal in terms of built-in equality, because a rule of the form

equal $(\mathrm{X}, \mathrm{Y}):-\mathrm{X}=\mathrm{Y}$

is not safe. As a consequence, such custom equality cannot be used in conjunction with built-in equality when computing a model, and thus rule (2) above escapes our mapping model.

Such rule, however, can be expressed as a constraint. Syntactically, DLV constraints are headless rules of the form

$$
:-L_{1}, \ldots, L_{n}
$$

The models of a program $P$ with such a rule added to it are the models of $P$ that do not satisfy $L_{1}, \ldots, L_{n}$. In other words, no model results from a program where the body of the constraint is satisfied. This can be used to express the anti-symmetry property above as a constraint, stating that there cannot be two distinct entities which are each the specialization of the other:

:- specializationOf $(\mathrm{E} 3, \mathrm{E} 2)$, specializationOf $(\mathrm{E} 2, \mathrm{E} 3)$, E2 $!=\mathrm{E} 3$ 
DLV enforces constraints and will signal that no model can be found for a given EDB. While this programming approach does not lead to the introduction of new nodes in provenance graphs, it provides a mechanism for checking the consistency of existing graphs with very limited programming effort. Examples of constraints in action are presented in Sec. 4. In the rest of the section we instead present examples of successful rule mappings along with associated provenance queries. A summary of all the rule mappings, including the constraints, indexed by the names given to the rules in Mor12b, appears in Table B in the Appendix.

\subsection{Examples Rules and Queries}

Inferring communication amongst activities. The following example illustrates how Datalog rules can be used to match patterns in the graph, and to find paths in the graph which connect instances of those patterns. Communication amongst activities is defined in [Mor12a as "the exchange of an entity by two activities, one activity using the entity generated by the other.". The wasInformedBy (informed, informant, attrs) relation is used to represent communication, where informant is the activity that provides an input entity to the informed activity. Mor12b states that a2 was informed by a1, if there is an entity e that has been generated by a1 and used by a2. Furthermore, Mor12b] also states that if e2 was derived from e1, and e2 was generated by activity a, then one can conclude that a used e1. These two rules are captured as follows:

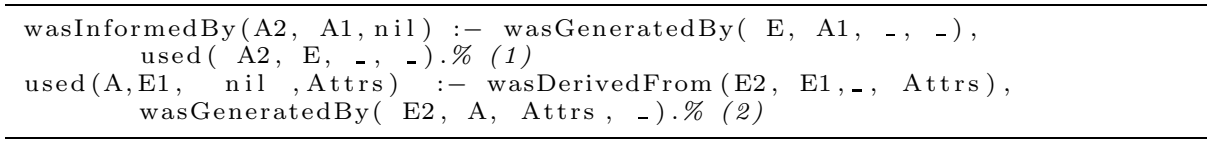

Each of these two rules capture a pattern in the provenance graph. As an example, used(editing, draftV1,nil), a relation that is not in the graph, is derived from (2), and from this, wasInformedBy (editing, drafting) also follows. From these, one can build upon these patterns by introducing further rules such as the following, which states that two agents are related through a path of length $n$, when they are associated to two activities, one of which is informed by the other. This provides an informal measure of "distance" amongst agents. The rule is recursive:

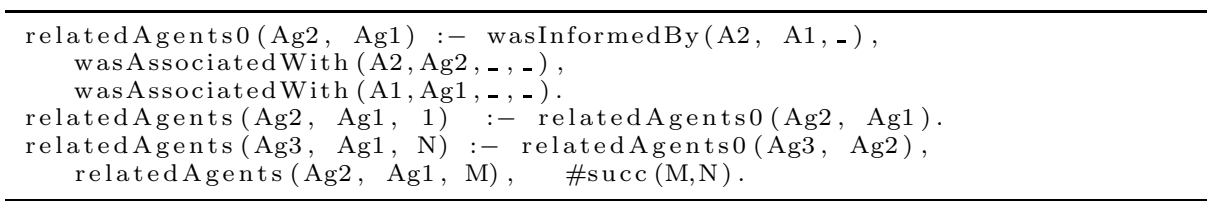

The built-in predicate $\# \operatorname{succ}(M, N)$ is true iff $\mathrm{N}==\mathrm{M}+1$. The query: 
relatedAgents $(\mathrm{Ag} 2, \operatorname{Ag} 1, \mathrm{~N})$ ?

returns, amongst others, the triple (charlie, bob_2, 1), which requires (1) and (2) for its derivation, and (charlie, bob_1, 3), which indicates that charlie and Bob are related by means of the entire chain of activities, from drafting to publication.

Agents' chains of responsibility. The next rule provides further illustration of the use of recursion, this time to state that an agent is ultimate responsibility for an activity, if either she is directly responsible or if another agent has acted on her behalf in the context of that activity 12 .

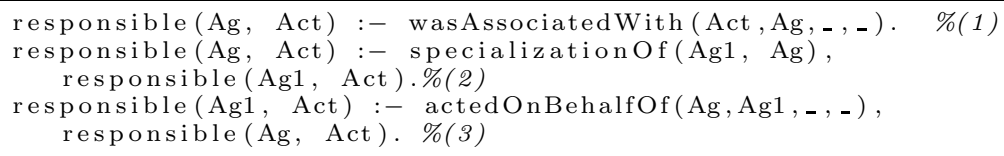

This reveals for example that alice is ultimately responsible for drafting, commenting, editing, and publishing. Finally, the following rule uses aggregation, a feature of DLV with an intuitive syntax, rather than recursion to determine that alice is at the head of a chain of responsibility (and so is, trivially, w3c_consortium):

headOfChain (Ag) :- was AssociatedWith (A, Ag, _, _ ), \#count $\left\{\right.$ Ag2: actedOnBehalfOf $\left.\left(\mathrm{Ag}, \mathrm{Ag} 2, \mathrm{~A}_{-}\right)\right\}=0$.

Entities with limited provenance. A final example of simple and potentially useful pattern query involves finding entities that have been used, but whose generation is unknown, making for "incomplete" provenance. The next rules makes use of aggregation for the purpose 13 :

ungenerated (E) :- used (, E, _, _ ),

\#count $\{\mathrm{A}: \quad$ wasGeneratedBy $(\mathrm{E}, \mathrm{A}, \ldots, \mathbf{-})\}=0$.

\section{Provenance Validation by Constraint Checking}

As anticipated in Sec. 3.2, constraints can be used in DLV as a way to map some of the PROV rules. Here we show constraints at work in two scenarios, both involving cycles in the graph. The first concerns temporal events, which are optionally associated with activities and most relations (Sec2). PROV defines temporal consistency by means of a number of event ordering rules, from the simplest: "the start of an activity precedes its end", to more involved ones:

$\overline{12}$ The additional literal specializationof $(\mathrm{Ag} 1, \mathrm{Ag})$ in $(2)$ is needed to associate responsibility to an agent in its abstract form.

13 Note that using negation-as-failure, a potentially more natural formulation, would result in an unsafe rule, i.e., ungenerated1 (E, T,Attrs) :- used(_, E, _, _), not wasGeneratedBy (E, A, T,Attrs). 
"if entity e is generated by a, then its generation time follows the start time of a". A provenance graph is temporally consistent if there exists a partial order amongst events, which satisfies all the temporal rules. Our encoding of these rules can be found in Appendix $\mathrm{B}$ and is based on the temporal precedence predicate precedes (T1, T2). Precedence is anti-symmetric and transitive:

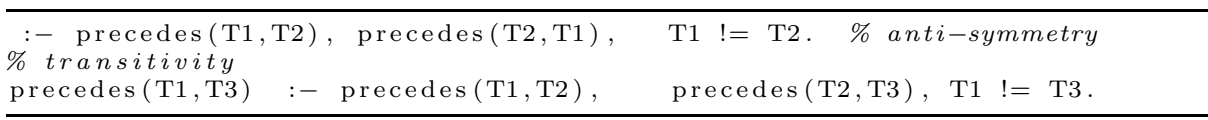

Query precedes $(\mathrm{T} 1, \mathrm{~T} 2)$ ? returns all partial order relations in the graph. In particular, temporal inconsistencies in the EDB are detected when no stable model is found. Fig. 2 shows an example of cyclic graph. Cycles in PROV graphs are in some cases acceptable, as shown in the temporal logic for the Open Provenance Model [KMV10]. Indeed in this example, the query returns a valid partial order (depicted in the bottom part of the figure). However, the program has no model when the precedence relation: precedes $\left(t \_u\right.$, a1Start) is explicitly added to the graph (not shown).

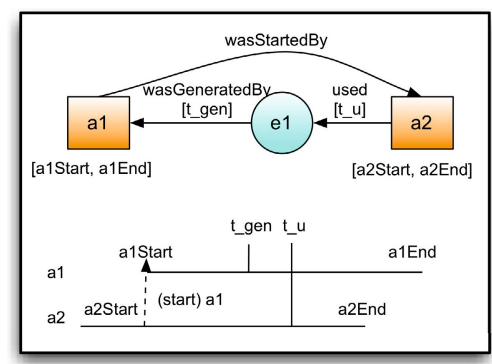

$$
\begin{aligned}
& \text { a1Start }<=\text { a2End } \\
& \text { a1Start }<=\text { t_u } \\
& \text { a1Start }<=\text { t_gen } \\
& \text { a2Start }<=\text { a1Start } \\
& \text { a2Start }<=\text { a1End } \\
& \text { a2Start }<=\text { t_u } \\
& \text { a2Start }<=\text { t_gen } \\
& \text { t_u }<=\text { a2End } \\
& \text { t_gen }<=\text { a1End } \\
& \text { t_gen }<=\text { a2End } \\
& \text { t_gen }<=\text { t_u }
\end{aligned}
$$

Fig. 2. Example of legal cycle in a PROV graph

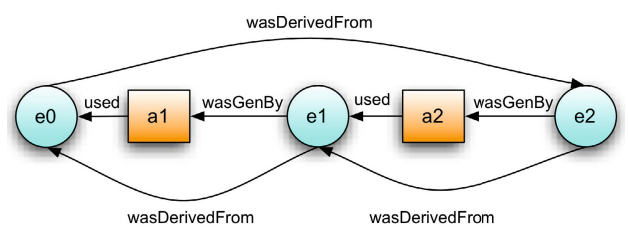

Fig. 3. Example of illegal cycle in a PROV graph

In contrast, some cycles in the provenance graph lead to inconsistencies, as in the example of Fig. 3. Here the cycle consisting of derivation relations is invalid, as derivation implies a time ordering amongst implicit use and generation events that accounts for it. Unless we accept to collapse all such events into one [KMV10], no order is possible.

The following rules are designed to detect these cycles: 


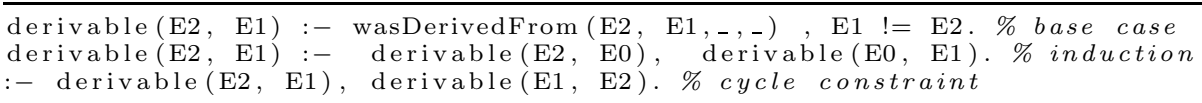

\section{Conclusions}

PROV is the emerging $\mathrm{W} 3 \mathrm{C}$ recommendation for a provenance data model and language. We have presented an encoding of PROV provenance graphs and inference rules and constraints as Datalog EDB and IDB, and we have shown how such encoding leads to intuitive, declarative-style queries on the graphs. Furthermore, we have used the constraint-solving capabilities of the DLV Datalog engine, which is freely available for non-commercial use, to show automated validation of PROV constraints, i.e., to detect temporal inconsistencies and illegal cycles in the provenance graph. An implementation of PROV-to-Datalog mapper is available online 14 , along with the complete set of Datalog rules and the examples used in the papen 15 .

In this work we have not addressed issues of efficient execution of queries on large graphs, a requirement that often conflicts with the declarative style of the query language, in the absence of suitable optimizations. Experiments are underway to test the limits of the DLV implementation. The potential uses in the provenance setting of disjunctive Datalog, which is typically used in automated planning applications, is also left for future research.

Acknowledgements. The authors would like to thank the members of the Provenance Working Group at the W3C for their collective effort on the PROV specification, and in particular Prof. Luc Moreau for contributing the PROV-toDatalog parser.

\section{References}

$\left[\mathrm{ABC}^{+} 10\right]$ Acar, U., Buneman, P., Cheney, J., Van Den Bussche, J., Kwasnikowska, N., Vansummeren, S.: A graph model of data and workflow provenance. In: Proceedings of the 2nd Conference on Theory and Practice of Provenance, TAPP 2010, p. 8. USENIX Association, Berkeley (2010)

[ABR10] Arenas, M., Barceló, P., Reutter, J.L.: Datalog as a Query Language for Data Exchange Systems. In: de Moor, O., Gottlob, G., Furche, T., Sellers, A. (eds.) Datalog 2010. LNCS, vol. 6702, pp. 302-320. Springer, Heidelberg (2011)

[ACG07] Atzeni, P., Cappellari, P., Gianforme, G.: Midst: model independent schema and data translation. In: SIGMOD Conference, pp. 1134-1136. ACM (2007)

[BET11] Brewka, G., Eiter, T., Truszczyński, M.: Answer set programming at a glance. Commun. ACM 54(12), 92-103 (2011)

${ }_{15} \begin{aligned} & \text { http://bit.ly/HOYJA8 } \\ & \text { http://bit.ly/HOY15T }\end{aligned}$ 
[CBD06] Cohen, S., Cohen-Boulakia, S., Davidson, S.B.: Towards a Model of Provenance and User Views in Scientific Workflows. In: Leser, U., Naumann, F., Eckman, B. (eds.) DILS 2006. LNCS (LNBI), vol. 4075, pp. 264-279. Springer, Heidelberg (2006)

[CGT89] Ceri, S., Gottlob, G., Tanca, L.: What you always wanted to know about Datalog (and never dared to ask). IEEE Transactions on Knowledge and Data Engineering 1(1), 146-166 (1989)

[CGT90] Ceri, S., Gottlob, G., Tanca, L.: Logic programming and databases. Springer-Verlag New York, Inc., New York (1990)

[DZL11] Dey, S.C., Zinn, D., Ludäscher, B.: ProPuB: Towards a Declarative Approach for Publishing Customized, Policy-Aware Provenance. In: Bayard Cushing, J., French, J., Bowers, S. (eds.) SSDBM 2011. LNCS, vol. 6809, pp. 225-243. Springer, Heidelberg (2011)

[EGM97] Eiter, T., Gottlob, G., Mannila, H.: Disjunctive datalog. ACM Trans. Database Syst. 22(3), 364-418 (1997)

[Hal01] Halevy, A.Y.: Answering queries using views: A survey. VLDB J. 10(4), 270-294 (2001)

[KMV10] Kwasnikowska, N., Moreau, L., Van den Bussche, J.: A Formal Account of the Open Provenance Model. Technical report, University of Southampton (December 2010)

[Mor12a] PROV-DM Part I: The PROV Data Model (March 2012), http://dvcs.w3.org/hg/prov/raw-file/default/model/prov-dm.html

[Mor12b] PROV-DM Part II: Constraints of the Provenance Data Model (March 2012), http://dvcs.w3.org/hg/prov/raw-file/default/ prov-dm-constraints.html

[Mor12c] PROV-DM Part III: The PROV Notation (March 2012), http://dvcs.w3.org/hg/prov/raw-file/default/model/prov-n.html

[SDNR07] Shen, W., Doan, A., Naughton, J.F., Ramakrishnan, R.: Declarative information extraction using datalog with embedded extraction predicates. In: VLDB, pp. 1033-1044. ACM (2007) 


\section{A Excerpts of the EDB for the Running Example}

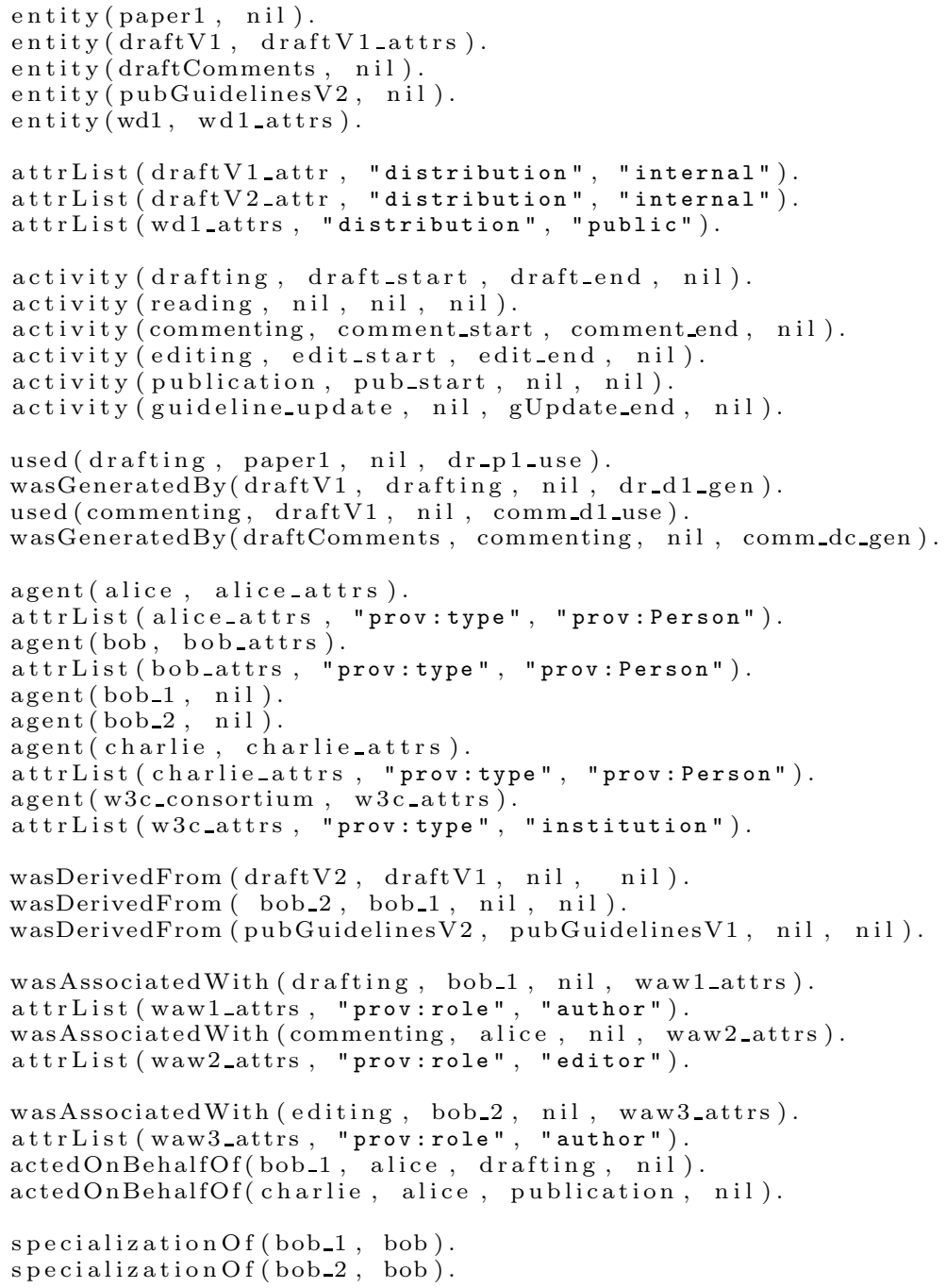




\section{B Summary of Datalog Rules Implemented for PROV}

\begin{tabular}{|c|c|}
\hline Constraint name & Datalog rule(s) \\
\hline Activities and Entities are disjoint & $:-\operatorname{activity}\left(\mathrm{X},,_{-},{ }^{\prime}\right)$, entity $\left(\mathrm{X},{ }_{-}\right)$. \\
\hline \multicolumn{2}{|c|}{ Event ordering interpretation constraints } \\
\hline Start of activity precedes its end & precedes (T1, T2) :- activity(_, _, T1, T2, _). \\
\hline $\begin{array}{l}\text { The generation of an entity always precedes } \\
\text { any of its usages }\end{array}$ & $\begin{array}{l}\text { precedes (T2,T1) :- used ( },, \text { E, _, T1), } \\
\text { wasGeneratedBy (E, _, -, T2). }\end{array}$ \\
\hline \multirow{2}{*}{ Usage-within-activity } & $\begin{array}{l}\left.\text { precedes }(\mathrm{T} 2, \mathrm{~T} 1):-\mathrm{used}_{\mathbf{1}}, \mathrm{E}, \ldots, \mathrm{T} 1\right), \\
\text { wasGeneratedBy }(\mathrm{E}, \ldots, \quad, \mathrm{T} 2) .\end{array}$ \\
\hline & $\begin{array}{l}\text { precedes (UT, T2) :- activity(A, _, T1, T2, - ), } \\
\text { used }\left(A_{-},-, \text {UT). }\right.\end{array}$ \\
\hline \multirow{2}{*}{ Generation-within-activity } & $\begin{array}{l}\text { precedes (T1, UT) :- activity(A, _, T1, T2, _), } \\
\text { used }\left(A_{-},-, \text {UT). }\right.\end{array}$ \\
\hline & $\begin{array}{l}\text { precedes(T1, GT) :- activity(A, _, T1, T2, -), } \\
\text { wasGeneratedBy (_, A,_, GT). }\end{array}$ \\
\hline Derivation-usage generation-ordering & $\begin{array}{l}\text { precedes }(\mathrm{T} 1, \mathrm{~T} 2) \text { :- wasDerivedFrom }(\mathrm{E} 2, \mathrm{E} 1, \mathrm{~A}, \ldots) \\
\text { used }(\mathrm{A}, \mathrm{E} 1,-, \mathrm{T} 2) \text {, wasGeneratedBy }(\mathrm{E} 2, \mathrm{~A},-, \mathrm{T} 1) .\end{array}$ \\
\hline \multirow[t]{2}{*}{ Detection of extended derivation loops } & $\begin{array}{l}:- \text { derivable(E2, E1), derivable(E1, E2). } \\
\text { derivable(E2, E1) :- wasDerivedFrom(E2, E1,-,-) } \\
\text { E1 != E2. }\end{array}$ \\
\hline & $\begin{array}{l}\text { derivable(E2, E1):- derivable(E2, E0), } \\
\text { derivable(E0, E1). }\end{array}$ \\
\hline derivation-generation generation-ordering & 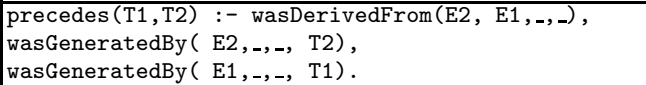 \\
\hline wasInformedBy-ordering & $\begin{array}{l}\text { precedes(ST1, ET2) :- wasInformedBy (A2, A1, }) \text {, } \\
\text { activity(A1, -, ST1, -, -), } \\
\text { activity (A2, _, _, ET2, _). }\end{array}$ \\
\hline wasStartedBy-ordering & $\begin{array}{l}\text { precedes }(\mathrm{ST} 1, \mathrm{ST} 2):- \text { wasStartedBy }\left(\mathrm{E} 2, \mathrm{E} 1,{ }_{-}\right), \\
\text {activity }\left(\mathrm{A} 1, \ldots, \mathrm{ST} 1,,_{-},{ }_{-}\right), \operatorname{activity}\left(\mathrm{A} 2, \ldots, \mathrm{ST} 2,,_{-}\right) .\end{array}$ \\
\hline $\begin{array}{l}\text { wasStartedByAgent-ordering, } \\
\text { wasAssociatedWith-ordering }\end{array}$ & $\begin{array}{l}\text { Not implemented because start and end events for } \\
\text { entities (and agents in particular) are not clearly de- } \\
\text { fined }\end{array}$ \\
\hline \multicolumn{2}{|c|}{ Structural constraints } \\
\hline Generation-uniqueness & $\begin{array}{l}:-\operatorname{activity}(\mathrm{A} 1,-,-,-,-), \text { activity }\left(\mathrm{A} 2,,_{-},,_{-}\right) \text {, } \\
\text { wasGeneratedBy }(\mathrm{E}, \mathrm{A} 1,-,-), \\
\text { wasGeneratedBy }\left(\mathrm{E}, \mathrm{A} 2,_{-},{ }_{-}\right), \mathrm{A}_{1} \mathrm{l}=\mathrm{A} 2 .\end{array}$ \\
\hline derivation-use & $\begin{array}{l}\text { used(A,E1, nil , Attrs) :- wasDerivedFrom(E2, E1, } \\
\text {-, Attrs), wasGeneratedBy( E2, A, Attrs, _). }\end{array}$ \\
\hline \multicolumn{2}{|c|}{ Element-specific constraints } \\
\hline Association-agent & $\begin{array}{l}\text { agent (E, Attrs) :- entity(E, Attrs), } \\
\text { wasAssociatedWith(_,E, , Attrs). }\end{array}$ \\
\hline Derivation-implication & $\begin{array}{l}\text { wasDerivedFrom(E2, E1, A, Attrs) :- } \\
\text { wasDerivedFrom(E2, E1, -, Attrs), wasGeneratedBy( } \\
\text { E2, A,_,_), used( A,E1,_,_). }\end{array}$ \\
\hline Transitivity of specialization & $\begin{array}{l}\text { specialization0f (E3,E1) :- } \\
\text { specialization0f (E3,E2), specialization0f (E2,E1). }\end{array}$ \\
\hline Anti-symmetry of specialization & $\begin{array}{l}\text { :- specialization0f(E1,E2), } \\
\text { specialization0f(E2,E1), E2 } !=\text { E1. }\end{array}$ \\
\hline Symmetry of alternate & $\begin{array}{l}:- \text { alternate0f }(\mathrm{E} 1, \mathrm{E} 2) \text {, alternate0f }(\mathrm{E} 2, \mathrm{E} 1), \text { E2 } != \\
\text { E1. }\end{array}$ \\
\hline Derivation implies traceability & tracedTo(E2, E1) :-wasDerivedFrom(E2,E1,_,_). \\
\hline Traceability of agent for a generating activity & $\begin{array}{l}\text { tracedTo(E2, Agent) :- -wasGeneratedBy( E2, A, _, _), } \\
\text { wasAssociatedWith (A, Agent,_.). }\end{array}$ \\
\hline $\begin{array}{l}\text { Traceability of a delegated agent for a gener- } \\
\text { ating activity }\end{array}$ & 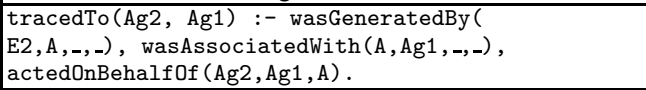 \\
\hline $\begin{array}{l}\text { Traceability by starting and generating activ- } \\
\text { ities }\end{array}$ & $\begin{array}{l}\text { tracedTo }(E 2, \text { E1) :-wasStartedBy }(\mathrm{A}, \mathrm{E} 1, \ldots) \text {, } \\
\text { wasGeneratedBy }\left(\mathrm{E} 2, \mathrm{~A},{ }_{-},{ }_{-}\right) .\end{array}$ \\
\hline Transitivity of traceability & $\begin{array}{l}\operatorname{tracedTo}(\mathrm{E} 2, \mathrm{E} 1):-\operatorname{tracedTo}(\mathrm{E} 3, \mathrm{E} 2), \\
\operatorname{tracedTo}(\mathrm{E} 2, \mathrm{E} 1) .\end{array}$ \\
\hline wasStartedBy (only 'if' part is actionable') & $\begin{array}{l}\text { wasStartedBy }(A 2, A 1):- \text { wasGeneratedBy }(E, A 1, \ldots) \text {, } \\
\text { wasStartedBy }(A 2, E, \ldots) \text {. }\end{array}$ \\
\hline
\end{tabular}

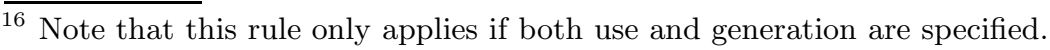

